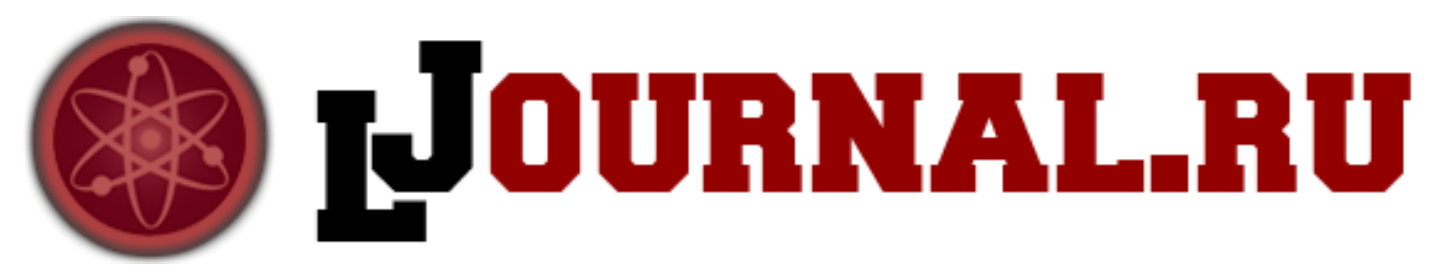

\author{
Пуга М.Ю., Гуськова О.Н. \\ ГБОУ ВПО Тверской ГМУ Минздрава России \\ Тверь, Россия
}

doi: 10.18411/1j2016-1-13

\title{
Аденома желудка: анализ эндоскопического биопсийного материала
}

Key words - biopsy, endoscopy, gastric adenoma, intestinal metaplasia, precancerous diseases.

Аннотация - важным аспектом превентивной медицины рака желудка является внедрение скрининговой диагностики предраковых изменений слизистой оболочки желудка, а также разработка методов их радикального лечения. Прицельная биопсия с последующим морфологическим исследованием позволяет с большой долей вероятности правильно оценить структуру патологического образования, a также выявить изменения, имеющие прогностическое значение. Благодаря наличию участков интраэпителиальной неоплазии аденома желудка является облигатным предраком. Цель исследования: изучение распространенности, локализации и особенностей морфологического строения аденомы желудка. В статье представлены результаты морфологического исследования 163 случаев аденомы желудка, отобранных из серии сплошного наблюдения гастробиоптатов 990 больных с полиповидными образованиями слизистой желудка. Проводилась оценка возраста, пола пациентов, локализации опухоли, ее размеров и морфологических особенностей. Результаты - аденомы желудка составили $16,46 \%$ от общего числа полиповидных образований слизистой оболочки желудка. Аденомы желудка имели одиночный характер и локализовались преимущественно в антральном 
отделе.Очаги тяжелой дисплазии были выявлены у 7 пациентов (4,3\%). Кишечная метаплазия, имеющая важное прогностическое значение, отмечена в 31 (19,02\%) аденоме. Ростакарциномывыявленонебыло.

ABSTRACT - The introduction of a screening diagnosis of precancerous lesions of gastric mucosa and elaboration of new methods of radical treatment is an important aspect of gastric cancer prevention. Digestive endoscopy with subsequent morphological study allows with high probability to correctly assess the structure of the lesion and to identify changes that have prognostic value. Gastric adenoma is the obligate precancerous lesion because the presence of intraepithelial neoplasia. Aim -to study of the stomach adenoma abundance, localization and morphological structure.Methods - 163 gastric adenomas were diagnosed by histological examination in a series of 990 patients who had upper digestive endoscopies done. The patients were analyzed as the age, sex, and the adenomas as the histological, as well as their location, size, histopathological findings studied. Results - gastric adenomas were diagnosed in $163(16,46 \%)$ in a series of patients with gasric polyps. Gastric adenomas were primarily single and developed in the antrum of stomach. High grade dysplastic foci were found in 7(4,3\%) adenomas, which size was lesser than 2 $\mathrm{cm}$. Intestinal metaplasia with high grade prognostic value was found in $31(19,02 \%)$ cases. Acarcinomawasnotdetected.

Введение: Рак желудка занимает 3-4 место в структуре онкологической заболеваемости россиян. Важным аспектом превентивной медицины рака является внедрение скрининговой диагностики предраковых состояний и изменений слизистой оболочки желудка[2]. Прицельная биопсия с последующим морфологическим исследованием позволяет с большой долей вероятности правильно оценить структуру патологического образования, а также выявить изменения, имеющие прогностическое значение [1]. В соответствии с международной гистологической классификацией опухолей морфологическим признаком аденомы желудка является наличие участков интраэпителиальной неоплазии, что позволяет расценивать ее как 
облигатныйпредрак[5]. По данным опубликованных исследований частота малигнизации аденомы желудка варьирует от 2,0\% до 30-40\% и коррелирует с размерами новообразования [3].

Целью исследования явилось изучение эпидемиологии и особенностей морфологического строения аденомы желудка.

Материалы и методы: из серии сплошного наблюдения гастробиоптатов патологоанатомического отделения ГБУЗ Тверской области «ОКБ» и клиникоморфологической лаборатории поликлиники ГБОУ ВПО «Тверской ГМУ Минздрава России» были отобраны гистологические препараты 990 пациентов с клиническим диагнозом «полип желудка». Аденома желудка была диагностирована в 163 случаев, что составило 16,46\% выборочной совокупности. Морфологическое исследование гистологических препаратов, окрашенных гематоксилином и эозином, проводили на микроскопе OLIMPUSCX-41. Дополнительные срезы с парафиновых блоков окрашивали альциановым синим в сочетании с ШИК-реакцией для идентификации гистохимического фенотипа кишечной метаплазии [4]. После гистологической верификации патологических изменений слизистой оболочки проведен клиникоморфологический анализ аденомы желудка. Оценивали возраст и пол пациентов, локализацию, морфологические особенности опухоли (размер, гистологический вариант строения, степень воспалительной инфильтрации, активность воспаления, тип кишечной метаплазии, категорию интраэпителиальной неоплазии). При обработке исходных данных применяли анализ произвольных таблиц сопряженности с оценкой статистической значимости различий по критерию $\chi^{2}$.

Результаты: Распределение пациентов по полу и возрасту показало, что у мужчин и женщин до 60 лет аденома желудка развивалась одинаково часто. Среди пациентов от 61 года и старше аденома желудка статистически значимо чаще встречалась у женщин.

Динамика заболеваемости по возрасту у мужчин и женщин представлена в 
таблице 1.

Таблица 1.

Возрастная структура больных с аденомами желудка, абс.(\%)

\begin{tabular}{|l|l|l|l|l|l|l|l|}
\hline Пол & $\begin{array}{l}\text { До 30 } \\
\text { лет }\end{array}$ & $\begin{array}{l}\mathbf{3 1 - 4 0} \\
\text { лет }\end{array}$ & $\begin{array}{l}\mathbf{4 1 - 5 0} \\
\text { лет }\end{array}$ & $\begin{array}{l}\mathbf{5 1 - 6 0} \\
\text { лет }\end{array}$ & $\begin{array}{l}\text { 61-70 } \\
\text { лет }\end{array}$ & $\begin{array}{l}\text { Старше } \\
\mathbf{7 0} \text { лет }\end{array}$ & Всего \\
\hline Мужчины & $4(2,45)$ & $6(3,68)$ & $9(5,52)$ & $9(5,52)$ & $11(6,75)$ & $13(7,98)$ & $52(31,90)$ \\
\hline Женщины & $4(2,45)$ & $5(3,07)$ & $12(7,36)$ & $24(14,72)$ & $25(15,34)$ & $41(25,15)$ & $111(68,10)$ \\
\hline
\end{tabular}

Изучение особенностей локализации показало, что у 112 (68,7\%) пациентов аденома желудка развивалась в антральном и пилорическом отделах. В теле желудка аденома была выявлена в 38 (23,3\%) наблюдениях, в кардиальном отделе - у 9 (5,5\%) больных, в области Z линии - у 4 (2,5\%) пациентов.

В 160 (98,2\%) наблюдениях полипы имели одиночный характер, у трех пациентов $(1,8 \%)$ выявлен диффузный полипоз. Макроскопически опухоли были представлены образованиями шаровидной, овальной и грибовидной формы размерами от 3 до 25 миллиметров.

В зависимости от особенностей гистологического строения были выявлены тубуло-папилярный, тубулярный, и папиллярный варианты аденомы. Распределение материала в соответствии с гистологическим вариантом и размерами новообразования представлено в таблице 2. По результатам исследования нам удалось выявить значимую связь между размером и гистологическим вариантом новообразования. Аденомы размером до 1 см, представлявшие собой образования на широком основании в виде бляшки или полусферы, преимущественно имели тубуло-папилярный вариант гистологического строения. 
Таблица 2.

\section{Распределение наблюдений в соответствии с гистологическим} вариантом и размерами новообразования, абс. (\%)

\begin{tabular}{|c|c|c|c|}
\hline \multicolumn{1}{|l|}{\begin{tabular}{|l|l|} 
Гистологический \\
Рубулиант
\end{tabular}} & $\begin{array}{l}\text { Тубулярный } \\
\text { папилярный }\end{array}$ & Папиллярный \\
\hline$<1 \mathrm{~cm}$ & $63(38,66)$ & $27(16,56)$ & $7(4,3)$ \\
\hline $1-2 \mathrm{~cm}$ & $34(20,86)$ & $16(9,81)$ & $2(1,22)$ \\
\hline$>2 \mathrm{~cm}$ & $6(3,68)$ & $7(4,3)$ & $1(0,61)$ \\
\hline Всего & $103(63,20)$ & $50(30,67)$ & $10(6,13)$ \\
\hline
\end{tabular}

Микроскопически новообразования были представлены очаговым скоплением железистых и сосочковых структур, располагающихся в пределах мышечной пластинки слизистой оболочки. В половине полипов размером более 2 см при микроскопическом исследовании были выявлены дефекты покровного эпителия в виде неглубоких эрозий, локализующихся на вершинах сосочковых выростов. В подлежащей ткани отмечалась выраженная смешанно-клеточная воспалительная инфильтрация и молодая грануляционная ткань. Повреждения поверхностного эпителия могут быть связаны с воспалением в ответ на травматизацию и периодические нарушения кровообращения.

Изучение очагов интраэпителиальной неоплазии показало отсутствие связи между степенью неоплазии и частотой встречаемости в данных гистологических вариантах. Данные представлены в таблице 3.

В $130(79,8 \%)$ эпителиальных новообразованиях была выявлена воспалительная инфильтрация стромы, представленная преимущественно клетками лимфоплазмоцитарного ряда и макрофагами с наличием небольшого числа нейтрофильных лейкоцитов. Выраженность воспалительной реакции коррелировала со степенью дисплазии эпителия. 
Таблица 3.

Выявление интраэпителиальной неоплазии в разных гистологических вариантах аденомы, абс. (\%)

\begin{tabular}{|l|l|l|l|}
\hline Категория ИЭН & Слабовыраженная & Умеренно выраженная & Тяжелая \\
$\begin{array}{l}\text { Гистоло- } \\
\text { гический } \\
\text { вариант аденомы }\end{array}$ & & & \\
\hline Тубуло-папилляный & $39(23,92 \%)$ & $58(35,58 \%)$ & $6(3,69 \%)$ \\
\hline Тубулярный & $18(11,04 \%)$ & $31(19,02 \%)$ & $1(0,61 \%)$ \\
\hline Папиллярный & $4(2,45 \%)$ & $6(3,68 \%)$ & \\
\hline Всего & $61(37,42 \%)$ & $95(58,28 \%)$ & $7(4,3 \%)$ \\
\hline
\end{tabular}

Появление признаков кишечной метаплазии свидетельствует об изменении дифференцировки камбиальных клеток эпителия под влиянием патологических воздействий. Формирование участков по типу неполной незрелой эпителиальной метаплазии является маркером высокого риска малигнизации. В исследованном нами материале кишечная метаплазия была выявлена в 31(19,02\%) биоптатов, из них I тип (полная) был отмечен в 15 (9,2\%) наблюдениях, II тип (неполная) - 15 (9,2\%), III тип (неполная толстокишечная) 1 (0,62\%). Данные представлены в таблице 4.

Полученные результаты позволяют предполагать, что морфологические изменения в ткани аденомы, в частности категория интраэпителиальной неоплазии, а также наличие и тип кишечной метаплазии, соответствуют стадии, на которой выявлено новообразование.

\section{Выводы:}

1. У мужчин и женщин в возрасте до 60 лет аденома желудка развивается одинаково часто и локализуется преимущественно в антральном или пилорическом отделе. Среди пациентов старше 60 лет аденома желудка чаще встречается у лиц женского пола.

2. Среди образований размером до 1 см преобладал тубуло-папиллярный 
гистологический вариант аденомы.

3. В аденоме тубуло-папиллярного строения с очагами слабой и умеренно выраженной интраэпителиальной неоплазии наиболее часто была выявлена зрелая метаплазия эпителия.

Таблица 4.

Изучение участков кишечной метаплазии эпителия в различных гистологических вариантах аденомы желудка, абс. (\%)

\begin{tabular}{|c|c|c|c|}
\hline \multirow{2}{*}{$\begin{array}{l}\text { Гистологический } \\
\text { вариант аденомы } \\
\text { желудка }\end{array}$} & \multicolumn{3}{|c|}{ Кишечная метаплазия } \\
\hline & $\begin{array}{l}\text { I тип (зрелая } \\
\text { КМ) }\end{array}$ & $\begin{array}{l}\text { II тип(неполная } \\
\text { КМ) }\end{array}$ & $\begin{array}{l}\text { III } \\
\text { тип(неполная } \\
\text { незрелая КМ) }\end{array}$ \\
\hline Тубуло-папиллярный & $10(6,14 \%)$ & $8(4,9 \%)$ & $1(0,62 \%)$ \\
\hline Тубулярный & $4(2,45 \%)$ & $5(3,07 \%)$ & - \\
\hline Папиллярный & $1(0,61 \%)$ & $2(1,23 \%)$ & - \\
\hline Всего & $15(9,2 \%)$ & $15(9,2 \%)$ & $1(0,62 \%)$ \\
\hline
\end{tabular}

\section{Литература}

1. В.Н. Сотников, Дубинская Т.К., Волова А.В., Есенин Д.Ю. Значение макроскопических признаков в диагностике и прогнозе морфологического типа полипа. // Клиническая медицина. Выпуск №2., 2011г. С.43-45.

2. Морфологическая диагностика болезней желудка и кишечника /Л. И. Аруин [и др.]; под общ.ред. Л. И. Аруина. - М.:Триада-Х, 1998. - 249 с.

3. Management of gastric polyps: an endoscopy-based approach/ Yasser H. Shaib. MassimoRugge. DavidY. Graham. AndRobertM. Genta/2013 
4. Management of gastric polyps:a pathology based guide for gastroenterologists/ Susanne W. Carmack, Robert M. Genta, David Y. Graham and Gregory Y. Lauwers/2009

5. Tumours of the Digestive System Edited by Stanley R. Hamilton Lauri A. Aaltonen-IARC Press - Lyon, 2000. - 36 c. 